\title{
Suicidio en Bogotá: un fenómeno que aumenta en poblaciones jóvenes
}

\author{
Ricardo Sánchez ${ }^{1}$, Silvia Orejarena ${ }^{2}$, Yahira Guzmán ${ }^{2}$, Janeth Forero ${ }^{3}$ \\ ${ }^{1}$ Departamento de Psiquiatría y Centro de Epidemiología Clínica, Facultad de Medicina, Universidad Nacional \\ de Colombia, Bogotá, D.C., Colombia. \\ 2 Departamento de Psiquiatría, Universidad Nacional de Colombia, Bogotá, D.C., Colombia. \\ ${ }^{3}$ Centro de Referencia Nacional sobre Violencia, Instituto Nacional de Medicina Legal y Ciencias Forenses, \\ Bogotá, D.C., Colombia.
}

Para analizar los patrones bimodales de la edad en víctimas de suicidio de Bogotá en tres puntos de corte, 1990, 1995 y 2000, se llevó a cabo un estudio observacional analítico con base en los expedientes del Instituto Nacional de Medicina Legal analizados mediante aplicación de modelos estadísticos de distribuciones de mezcla finita. Se analizaron 222 suicidios en 1990, 272 en 1995 y 330 en el 2000. Se comprobó la presencia de un patrón bimodal en cada uno de los años analizados. Las medias de cada uno de los componentes responsables de las dos modas se hicieron progresivamente menores con el transcurso del tiempo. En Bogotá se dio una distribución bimodal en la edad de los suicidas a lo largo del período analizado. La tendencia de las medias sugiere que, con el transcurso del tiempo, los suicidios en Bogotá tienden a ocurrir en personas más jóvenes. Este fenómeno debe ser objeto de investigación adicional y de aplicación de estrategias específicas en salud mental dirigidas a estos grupos en riesgo.

Palabras clave: suicidio, grupos por edad, factores de riesgo.

\section{Suicide in Bogota: a growing phenomenum among young populations}

An observational analytic study was carried out to analyze bimodal patterns related with age in suicidal victims in Bogotá during 1990, 1995 and 2000 using information from autopsy reports. The analysis was based on statistical models of finite mixture distributions, and parameters were estimated with maximum likelihood methods. Two hundred and twenty two suicides were analyzed in 1990, 272 in 1995, and 330 in 2000. The presence of a bimodal distribution in each one of the analyzed years was demonstrated. As time passes, the means became progressively smaller. Age showed a bimodal distribution during the analyzed period. Means tendencies suggest that suicide is becoming more frequent among young people. Additional research and specific mental health strategies are recommended for these risk groups.

Key words: suicide, age groups, risk factors.

Según la Organización Mundial de la Salud, se estima que en el año 2000, aproximadamente, un millón de personas murieron por suicidio (1). Esto implica que cada 40 segundos hay un suicida en algún lugar del mundo y que cada tres segundos una persona intenta suicidarse (2). Mundialmente, el suicidio es una de las cinco causas de

Correspondencia:

Ricardo Sánchez, Oficina 202, Facultad de Medicina, Bogotá. Teléfono 316 5000, extensión 15 117; fax: 2428500 risanche@bacata.usc.unal.edu.co

Recibido: 04/07/02; aceptado: 15/11/02 mortalidad en la franja de edad entre los 15 y los 19 años y en muchos países se ubica como la primera o segunda causa de muerte tanto en varones como en mujeres en este grupo de edad (3). El suicidio está entre las primeras 10 causas de muerte en muchos países y es una de las tres primeras causas de muerte en el grupo de edad de 15 a 35 años. En Bogotá es la tercera causa de muerte violenta y ha dejado 1.642 víctimas en los últimos cinco años (4). Para el 2000, en esta ciudad se estimó una tasa de 5,4 por 100.000 hab y se encontró el $50 \%$ de las víctimas con edades entre los 11 y los 28 años de edad (5). 
Esta frecuencia es similar a la reportada a nivel nacional (5,0 por 100.000) y se ubica en un punto intermedio entre regiones con mayor frecuencia (9,4 por 100.000 en Quindío) y regiones con incidencia muy baja $(0,5$ por 100.000 en Chocó) (6).

El suicida produce un importante impacto psicológico en su familia y en su grupo social (en promedio, un suicida afecta cercanamente a, por lo menos, 6 personas). El número de afectados se incrementa de manera importante si se tiene en cuenta el entorno académico y social de la persona. La carga que produce el suicidio puede estimarse en términos de años de vida ajustados por discapacidad (DALY). Según este indicador, en 1998, el suicidio fue responsable del $1,8 \%$ de la carga total de la enfermedad a nivel mundial, variando entre 2,3\% en los países de altos ingresos y $1,7 \%$ en los de bajos ingresos. En los últimos 45 años, la mortalidad por esta causa se ha incrementado en un $60 \%$, tendencia observada tanto en los países desarrollados como en los en vías de desarrollo.

Aunque varios autores han descrito que el suicidio es un fenómeno característico de los extremos de edad (7), especialmente en países desarrollados (8-10), en las últimas cuatro décadas las mayores tasas de suicidio se han desplazado desde los grupos de edades altas a las personas más jóvenes (35 y 45 años y aún 15 a 25 años en algunos sitios) (11). En los últimos años se ha evidenciado que la tasa de suicidios y parasuicidios aumentó hasta en un 30\% en el grupo de varones jóvenes, especialmente entre los 25 y los 34 años de edad (12).

Varios factores se han propuesto para explicar la relación observada entre edad y suicidio: para algunos, el acceso a las armas de fuego, especialmente en hombres y en edades cada vez más tempranas, facilita la ejecución de conductas suicidas, ya que en el grupo de edad de 5 a 14 años el tener acceso a tal tipo de armas aumenta hasta 7 veces los suicidios (13).

Otro factor que puede influir en la relación del suicidio con la edad es el cambio en la dinámica familiar y el aumento del número de personas separadas, pues se ha visto que son mayores las tasas de suicidio entre estas personas que entre las casadas o viudas (14).

También se ha implicado el estado de salud, tanto física como mental, ya que son más frecuentes los suicidios entre personas con enfermedades mentales, especialmente las de tipo afectivo, consumo de alcohol y sustancias, y trastornos de personalidad (15-17); estas entidades se presentan con mayor frecuencia en personas jóvenes.

Un determinante de gran importancia se relaciona con los aspectos socioeconómicos: el aumento del índice de desempleo, la violencia, el aumento de la población de bajo nivel socioeconómico, la fragmentación social y el fácil acceso a métodos letales son factores que indirectamente llevan al aumento de la tasa de mortalidad y en especial de la relacionada con el suicidio (18-20), siendo esta situación más evidente en los hombres jóvenes que son los más afectados y los más sensibles a los cambios sociodemográficos (21).

Debe destacarse el impacto que tienen los medios de comunicación en las conductas suicidas, particularmente en jóvenes. Una de las primeras relaciones entre medios de comunicación y suicidio se observó con la publicación, en 1774, de la novela de Goethe, Die Leiden des jungen Werter, que generó un número alto de suicidas imitando al protagonista de la obra. Dado este antecedente se acuñó el término 'efecto Werter' para referirse a los suicidios por imitación (22). Algunos autores plantean que el grado de publicidad que se dé a una historia de suicidio se relaciona directamente con el número de suicidios posteriores, especialmente si se trata del caso de una celebridad o personaje importante (23). Philips encontró que la frecuencia de suicidios aumentaba hasta 10 días después de la información televisada sobre casos de suicidio (24).

En este estudio se busca determinar si, a lo largo del tiempo, existe un patrón bimodal en la distribución de la edad de las víctimas de suicidio en Bogotá y evaluar el comportamiento de ese patrón, tomando para esto tres puntos de corte en un período de una década: años 1990, 1995 y 2000. 


\section{Materiales y métodos}

El presente estudio se realizó tomando como base los expedientes o archivos físicos (actas de inspección de cadáver, protocolos de necropsias, dictámenes de laboratorio) del Instituto Nacional de Medicina Legal de Bogotá de los años 1990, 1995 y 2000. Del total de necropsias efectuadas en esos años, se seleccionaron aquellos casos con confirmación forense de muerte suicida ocurrida en la jurisdicción de la capital. Para cada uno de los casos que cumplieron este criterio de inclusión se registró la edad que aparecía anotada en el protocolo de necropsia.

El análisis de los patrones bimodales de la variable edad se efectuó con base en el concepto estadístico de distribuciones mezcladas. Este tipo de distribuciones puede usarse para el modelo de los datos correspondientes a poblaciones en las que se sospecha que exista un número de subpoblaciones separadas (25). Para tal efecto, se plantea una función de densidad de probabilidad mixta, que es una suma ponderada de $\mathrm{k}$ densidades que la componen.

Se utilizó un modelo de distribución normal mezclada, en el cual los parámetros que se utilizaron fueron los valores de probabilidades de mezcla, $p$, las medias $\mu$ y las desviaciones estándar o de las plausibles distribuciones componentes en cada uno de los años estudiados (26). Dichos parámetros se estimaron con métodos de máxima verosimilitud (27), usando el algoritmo modificado de Newton-Raphson (28). Los procedimientos estadísticos se efectuaron con el programa Stata7 (29).

Con el fin de establecer si las dos medias estimadas por el método anterior en cada uno de los años estudiados corresponden a diferentes poblaciones, se utilizó la prueba de Wolfe (30). De acuerdo con esta prueba, la diferencia en menos dos veces el log -verosímil para un modelo que tenga $c$ componentes, en comparación con un modelo con $c+1$ componentes- presenta una distribución ji cuadrado con $2 v-2$ grados de libertad, siendo $v$ el número de parámetros que se estiman en el componente mezclado $c+1$.

Desde el punto de vista ético, y de acuerdo con la legislación colombiana, ésta es una investigación sin riesgo, ya que no se realiza ninguna intervención ni modificación intencionada de variables biológicas, fisiológicas, sicológicas 0 sociales en seres humanos (31). En todo momento se garantizó la privacidad de los sujetos implicados en esta investigación.

\section{Resultados}

El número de casos de suicidio en cada año, así como las características de la variable edad en cuanto a media, desviación estándar, mediana y rango se muestran en el cuadro 1.

Se efectuó una exploración gráfica preliminar utilizando un histograma; el número óptimo de etapas de esta herramienta gráfica se determinó mediante la función propuesta por Emerson y Hoalgin (32) para muestras grandes, donde $n$ es el número total de observaciones.

Los histogramas para cada uno de los años se muestran en las figuras 1, 2 y 3.

La estructura de los histogramas insinúa la presencia de dos componentes mezclados que, en las distribuciones de cada uno de los tres años tienen: uno, media alrededor de los 25 años y otro, media cercana a los 45 años. En los tres años se perfila una mayor variabilidad en el componente con media mayor.

Para ejecutar los procedimientos de máxima verosimilitud y teniendo en cuenta los valores sugeridos gráficamente por los histogramas, se

Cuadro 1. Número de suicidios y características de la edad de suicidas, Bogotá, 1990, 1995 y 2000.

\begin{tabular}{cccccc}
\hline Año & $\begin{array}{c}\text { Número de } \\
\text { suicidios }(\mathbf{n})\end{array}$ & $\begin{array}{c}\text { Media de la edad } \\
\text { de los suicidas }\end{array}$ & $\begin{array}{c}\text { Desviación } \\
\text { estándar }\end{array}$ & $\begin{array}{c}\text { Mediana de la edad } \\
\text { de los suicidas }\end{array}$ & $\begin{array}{c}\text { Rango de edad } \\
\text { de suicidas (años) }\end{array}$ \\
\hline $\mathbf{1 9 9 0}$ & 222 & 35,5 & 15,9 & 32 & 11 a 82 \\
$\mathbf{1 9 9 5}$ & 272 & 31,1 & 15,2 & 26 & 10 a 86 \\
$\mathbf{2 0 0 0}$ & 330 & 32,6 & 14,7 & 28 & 11 a 77 \\
\hline
\end{tabular}




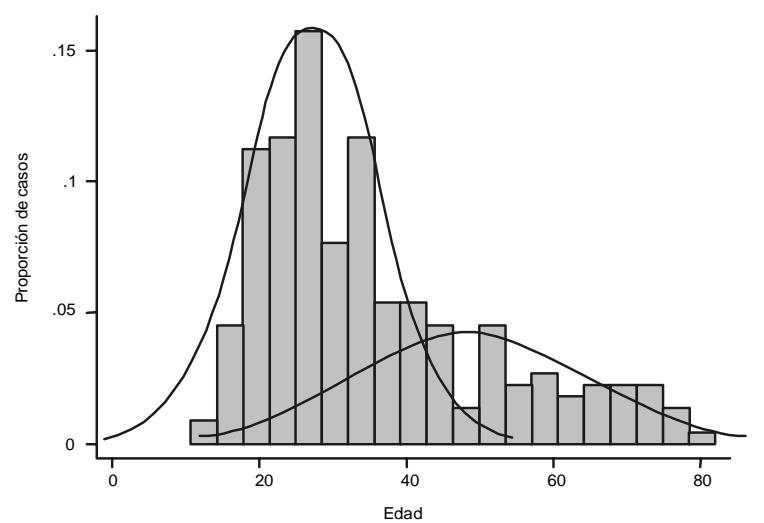

Figura 1. Histograma de la edad de suicidas, Bogotá, 1990.

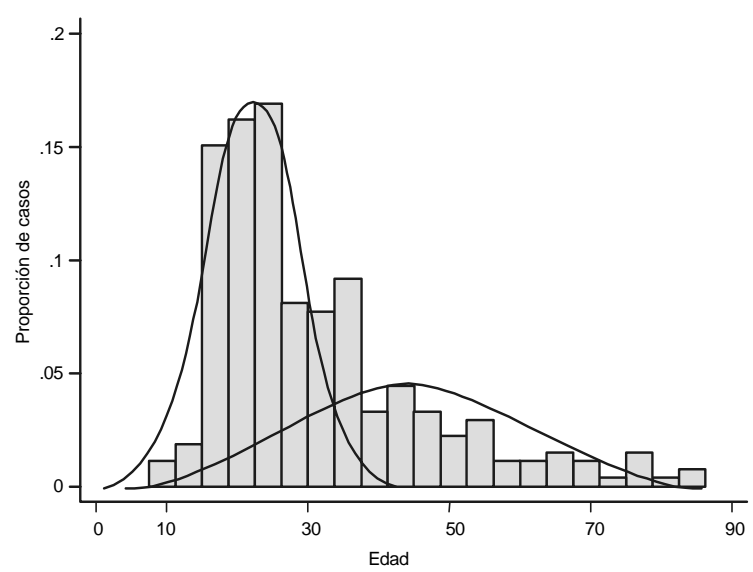

Figura 2. Histograma de la edad de suicidas de Bogotá, 1995.

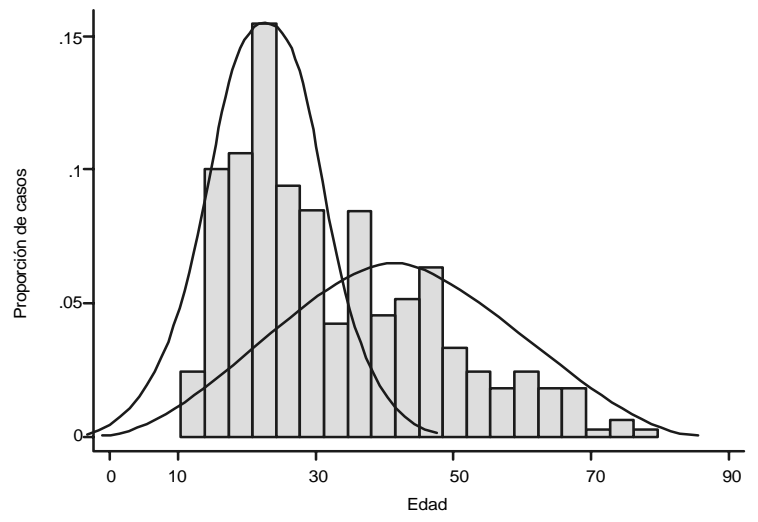

Figura 3. Histograma de la edad de suicidas de Bogotá, 2000. construyó una matriz de valores iniciales para las iteraciones con los siguientes datos: media de la distribución $a=25$; media de la distribución $b=45$; desviación estándar de la distribución $a=10$, y desviación estándar de la distribución b=20.

Para 1990, el procedimiento de máxima verosimilitud muestra la presencia de dos componentes dentro de la distribución inicialmente mezclada: uno con una media de 27 años y otro con media de 53,2 años. El primer componente muestra una variabilidad menor y un mayor número de observaciones, mientras que el segundo componente corresponde a un grupo más pequeño y más heterogéneo (cuadro 2). Al aplicar la prueba de Wolfe se encontró que las medias de los componentes corresponden a diferentes poblaciones $\left(\chi_{(4)}^{2}=75,75, P=0,000\right)$.

De manera similar, en 1995 se encontraron dos componentes mezclados con medias de 22,46 y 43,67 años. Al igual que en 1990, el segundo componente tiene mayor variabilidad. Ambos componentes tienen un número similar de observaciones (cuadro 2). La prueba de Wolfe mostró que las medias de los componentes corresponden a diferentes poblaciones $\left(\chi^{2}{ }_{(4)}=117,02, P=0,000\right)$. De igual forma que en los años anteriores, en 2000 se identifican dos componentes mezclados cuyas medias son 21,33 y 42,36 , respectivamente. El componente con la media mayor sigue teniendo mayor variabilidad. Tal y como sucedió en los años anteriores, la prueba de Wolfe indicó que las dos medias corresponden a diferentes poblaciones $\left(\chi_{(4)}^{2}=95,99, P=0,000\right)$.

La tendencia de los diferentes valores de las medias en los diferentes años estudiados se muestra en la figura 4. Como puede verse, a medida que transcurre el tiempo, las medias de cada uno de los grupos de los respectivos años tienen tendencia a ser menores.

\section{Discusión}

Los resultados de este estudio sugieren que en Bogotá existe un patrón bimodal en la edad de las víctimas de suicidio y que este patrón se presenta desde hace varios años. El resultado del estudio contrasta con algunos reportes de la literatura de países industrializados, donde se 
Cuadro 2. Características de la variable edad en cada uno de los componentes mezclados, 1990, 1995 y 2000.

\begin{tabular}{lcccccrrr}
\hline Año & Componente & Media & $\begin{array}{c}\text { Desviación } \\
\text { estándar }\end{array}$ & Intervalos de confianza 95\% & Proporción & $\#$ \\
\hline 1990 & 1 & 27,0 & 7,1 & 24,94 & 29,05 & 0,67 & 149 \\
& 2 & 53,2 & 14,1 & 43,73 & 62,74 & 0,33 & 73 \\
1995 & 1 & 22,5 & 5,5 & 20,78 & 24,13 & 0,59 & 161 \\
& 2 & 43,7 & 16,0 & 38,09 & 49,24 & 0,41 & 111 \\
2000 & 1 & 21,3 & 4,9 & 20,17 & 22,49 & 0,46 & 152 \\
& 2 & 42,4 & 13,2 & 38,98 & 45,73 & 0,54 & 178 \\
\hline
\end{tabular}

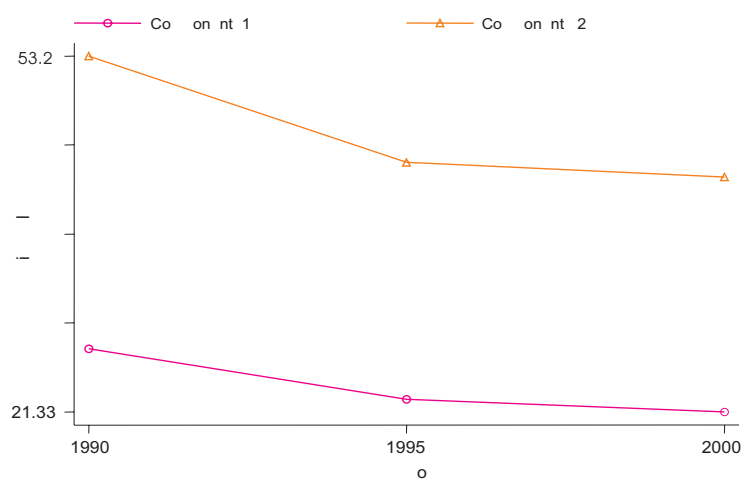

Figura 4. Comportamiento de la media de edad para cada componente.

encuentran medias por encima de 65 años en la moda mayor $(33,34)$, relacionadas con frecuencia más alta de trastornos depresivos y con particularidades de eventos vitales como viudez y aislamiento social (35-37). Sin embargo, este estudio también apoya la tendencia reportada en la literatura mundial, en relación con el progresivo aumento de conductas suicidas y parasuicidas en jóvenes. Como se planteó en un estudio previo (38), el hecho de encontrar una moda mayor con edades más bajas que las reportadas en algunos países desarrollados parece ser una característica de la población de Bogotá que refleja una dinámica mundial, ya que se mantiene en los tres puntos de corte evaluados: 1990, 1995 y 2000. En estudios posteriores debe estudiarse la posibilidad de que dicha característica sea compartida por otras ciudades de países no industrializados, lo cual sugeriría la presencia de determinantes diferentes de acuerdo con las características propias de cada región.

El presente estudio también sugiere que las medias de los dos componentes, el de edad mayor y el de edad menor, tienden a reducirse a medida que transcurre el tiempo. Esto indicaría que cada vez hay personas más jóvenes entre las víctimas de suicidio en Bogotá, lo cual supone que el suicidio está generando una carga cada vez mayor en términos de años de vida ajustados por discapacidad (DALY).

La dinámica de reducción de las medias de edad de suicidas puede estar reflejando, entre otros aspectos, una disminución en la población de ancianos en Bogotá o un incremento de factores de riesgo en poblaciones de edad media y joven. El primero de estos aspectos (disminución de población vieja) podría descartarse si se observa la tendencia de la población en los mayores de 65 años en Bogotá: de acuerdo con los datos del Departamento Nacional de Planeación (39), la población de mayores de 65 años en esta ciudad era de 223.567 personas en 1995 y aumentó a 273.110 en el 2000. Teniendo en cuenta lo anterior, consideramos que en Bogotá existen factores de riesgo que están ejerciendo un efecto aún no evaluado y están generando una tendencia a que el suicidio se presente cada vez más en poblaciones jóvenes.

Como previamente se mencionó, se ha reportado que los desencadenantes de tipo económico y ocupacional son factores de riesgo importantes, especialmente en grupos de personas con vida laboral activa e, incluso, en adolescentes (40). Es posible que la dinámica observada en el presente estudio esté reflejando la situación de crisis social y económica que atraviesa Colombia desde hace varios años. Otro aspecto que se debe investigar en estudios posteriores se relaciona con la frecuencia de enfermedades mentales y su distribución según la edad. Se podría pensar que 
el aumento de suicidios en jóvenes se relacione con una mayor frecuencia de enfermedades mentales en esta población. Se ha informado que más del $10 \%$ de los casos de depresión se presenta entre los 14 y los 16 años (41) y que una de las complicaciones más frecuentes en pacientes con enfermedades afectivas es el suicidio $(42,43)$. En este sentido, también debe destacarse la relación entre el uso patológico de sustancias y el suicidio que se ha descrito en poblaciones de adolescentes (44-47). Otro aspecto que debe tenerse en cuenta es la relación entre eventos vitales y conductas suicidas, referida especialmente en adolescentes con enfermedad mental (48). Tan importante es el papel de la salud mental que en algunas series se ha detectado que unos de los mejores predictores de suicidio son el haber requerido hospitalización psiquiátrica, especialmente si se es de clase social alta (49) y se han tenido intentos previos (50).

Resulta especialmente importante estudiar el papel que tiene el acceso a métodos letales de suicidio por parte de los jóvenes y la participación de los medios de comunicación como eventuales facilitadores o promotores de conductas suicidas, dada la posibilidad de aplicar medidas preventivas a este nivel. Es tal la importancia de este aspecto que la OMS ha desarrollado material dirigido a los profesionales de los medios de comunicación con el fin de prevenir muertes por esta causa (51).

Debe reconocerse que la mayor utilidad de un estudio sobre el suicidio se relaciona con la ubicación de los factores de riesgo, determinantes o precipitantes, sobre los cuales se puedan ejecutar acciones específicas que se reflejen en medidas preventivas reales. Infortunadamente, las fuentes de información utilizadas en el presente estudio (actas de inspección de cadáver, protocolos de necropsias, dictámenes de laboratorio) no permiten una aproximación adecuada para analizar patrones de causalidad ya que la información relacionada con este aspecto en muchos casos no se recolecta y su registro tampoco se deriva de un proceso sistemático de recolección de tales datos. La aproximación a los posibles factores causales supone estudios prospectivos en los cuales se apliquen estrategias de recolección sistemática de información a los familiares de los suicidas (autopsias psicológicas), o se utilicen entrevistas estructuradas o escalas específicas a pacientes sobrevivientes de intentos graves de suicidio.

Teniendo en cuenta que en los casos de muerte violenta la práctica de la autopsia médico-legal es obligatoria, se reduce la posibilidad de subregistro de casos, razón por la cual consideramos que los estimadores encontrados pueden estar reflejando razonablemente los parámetros de la población.

Como recomendación práctica en relación con la evaluación de riesgo suicida en pacientes en Bogotá, debe tenerse en cuenta que las edades de mayor riesgo están alrededor de los 25 y los 45 años.

\section{Agradecimientos}

Este estudio contó con apoyo financiero de la División de Investigación de Bogotá de la Universidad Nacional de Colombia (809216).

\section{Referencias}

1. World Health Organization. Preventing suicide. A resource for general physicians. Geneva: World Health Organization; 2000. p.5.

2. World Health Organization. Preventing suicide. A resource for primary health care workers. Geneva: World Health Organization; 2000. p.5-6.

3. Organización Mundial de la Salud. Prevención del suicidio. Un instrumento para docentes y demás personal institucional. Ginebra: Organización Mundial de la Salud; 2001. p.1.

4. Perdomo M, Caicedo C, Garavito A, Hernández UK, Schock C. Suicidio por asfixia mecánica - ahorcadura, Bogotá 1996 - 2000. Centro Nacional de Referencia sobre Violencia 2001;6.

5. Forero J, Pérez I. Exploración de algunas relaciones entre suicidio, alcohol y otras sustancias psicoactivas, Bogotá 2000. Centro Nacional de Referencia sobre Violencia $2001 ; 6$

6. Ministerio de Salud. Sistema Nacional de Vigilancia en Salud Pública. Semana Epidemiológica \# 26, junio 25 a julio 1 de 2000.

7. Mósciki EK. Epidemiology of suicidal behavior. Suicide Life Threat Behav 1995;25:22-35.

8. Mósciki EK. Identification of suicide risk factors using epidemiologic studies. Psychiatr Clin North Am 1997;20: 499-517. 
9. Jamison K, Baldessarini R. Effects of medical interventions on suicidal behavior. J Clin Psychiatry 1999;60:4-6.

10. Hirshfeld R, Rusell J. Assessment and treatment of suicidal patients. N Engl J Med 1997;337:910-5.

11. WHO. Multisite Intervention Study on Suicidal Behaviours: Components and Instruments. Geneva: Department of Mental Health and Substance Dependence, World Health Organization; 2000. p.1.

12. Jenkins ER, Geiffiths S, Wylie I, Hawton K, MorganG, Tylee A. The prevention of suicide. BMJ 1994;309:886.

13. Miller M, Azrael D, Hememway D. Firearm availability and Unintentional firearm deaths, Suicide, and homicide among 5-14 year olds. J Trauma 2002;52:267-75.

14. Cheng A, Chen T, Chen $\mathbf{C H}$, Jenkins R. Psychosocial and psychiatry risk factors for suicide. $\mathrm{Br} \mathrm{J}$ Psychiatry 2000;177:360-5.

15. Quin P, Mortensen PB, Agerbo E. Gender differences in risk factors for suicide in Denmark. $\mathrm{Br} \mathrm{J}$ Psychiatry 2000; $177: 546-50$

16. Hawton K. Sex and suicide. Br J Psychiatry 2000;177: 484-5.

17. Goldstein RB, Black DW, Nasrallah A, Winokur G. The prediction of suicide. Arch Gen Psychiatry 1991;48: 418-22.

18. Lewis G, Slogget A. Suicide, deprivation, and unemployment: record linkage study. BMJ 1998;317: 1283-6.

19. Pappas G, Queen S, Hadden W, Fisher G. The increasing Disparity Between Socioeconomic groups in the United States, 1960 and 1986. N Engl J Med 1993;329: 103-9.

20. Viilo K, Hakko H, Väisänen E, Räsänen P. Risk of suicide related to income level in mental illness. Letter. BMJ 2001;323:232.

21. Whitley E, Gunnel D, Dorling D, Smith GD. Ecological study of social fragmentation, poverty, and suicide. BMJ 1999;319:1034-7.

22. Jonas K. Modeling and suicide: a test of the Werther effect. British Psychological Society 1992;31:295-306.

23. Wasserman D. Imitation and suicide: a re-examination of the Werther effect. American Sociological Review 1984; 49:427-36

24. Philips DP. The impact of fictional television stories on US adult fatalities: new evidence on the effect of the mass media on violence. American Journal of Sociology 1982;87:1340-59

25. Everitt BS. An introduction to finite mixture distributions. Statistical Methods in Medical Research 1996;5:107-27.

26. Rabe-Hesketh S, Everitt B. A handbook of statistical analysis using Stata. Boca Raton: Chapman \& Hall; 1998. p.179-93.
27. Mood AM, Graybill FA, Boes DC. Introduction to the theory of statistics. Singapore: McGraw-Hill; 1974. p.122-4.

28. Gould W, Sribney W. Maximum likelihood estimation with Stata. College Station, Texas: Stata Press.

29. StataCorp. Stata Statistical Software: release 7.0. College Station, Texas: Stata Corporation; 2001.

30. Wolfe JH. A Monte Carlo simulation of the sampling distribution of the likelihood ratio for mixtures of multinormal distributions. STB72-2. Technical Bulletin, Naval personnel and training research laboratory; 1971.

31. Ministerio de Salud de Colombia. Resolución 8430 de Octubre 4 de 1993, artículo 11. Ministerio de Salud, Dirección de desarrollo científico y tecnológico, República de Colombia, Bogotá, DC.

32. Emerson JD, Hoalgin DC. Stem and leaf displays. En: Understanding robust and exploratory data analysis. Hoalgin F, Mosteller F, Tukey JW, editors. New York: John Wiley \& Sons; 1983. p.7-30.

33. Jamison K, Baldessarini R. Effects of Medical Interventions on Suicidal Behavior. J Clin Psychiatry 1999; 60:4-6.

34. Hirshfeld R, Rusell J. Assessment and Treatment of Suicidal Patients. N Engl J Medicine 1997;337:910-5.

35. Beck AT, Steer R. Clinical predictors of eventual suicide: a 5-to 10 year prospective study of suicide attempters. J Affect Disorders 1989;57:309-10.

36. Motto J. An integrated approach to estimating suicide risk. Suicide Life Threat Behav 1991;21: 74-89.

37. Truant G, O'Reily R, Donaldson L. How Psychiatrist Weigh Risk Factors When Assessing Suicide Risk. Suicide Life Threat Behav 1991;21:106-14.

38. Sánchez R, Guzmán Y, Orejarena S. Características de la edad en suicidas de Bogotá durante 1999 y 2000. Acta Méd Colomb 2000;27:115-8.

39. Departamento Nacional de Planeación. Sistema de indicadores sociodemográficos para Colombia. http:// www.dnp.gov.co/01_CONT/INDICADO/SISD.HTM\#4.

40. Fergusson DM, Horwood LJ, Woodward LJ. Unemployment and psychosocial adjustment in young adults: causation or selection? Soc Sci Med 2001;53:30520.

41. Fergusson DM, Woodward LJ.Mental health, educational, and social role outcomes of adolescents with depression. Arch Gen Psychiatry 2002;59:225-31.

42. Angst F, Stassen HH, Clayton PJ, Angst J. Mortality of patients with mood disorders: follow-up over 34-38 years. J Affect Disord 2002;68:167-81.

43. Coryell W, Haley J, Endicott J, Solomon D, Leon AC, Keller M, Turvey C, Maser JD, Mueller T.The prospectively observed course of illness among 
depressed patients who commit suicide. Acta Psychiatr Scand 2002;105:218-23.

44. Colby SM, Barnett NP, Eaton CA, Spirito A, Woolard R, Lewander W, Rohsenow DJ, Monti PM. Potential biases in case detection of alcohol involvement among adolescents in an emergency department. Pediatr Emerg Care 2002;18:350-4.

45. Kelly TM, Cornelius JR, Lynch KG. Psychiatric and substance use disorders as risk factors for attempted suicide among adolescents: a case control study. Suicide Life Threat Behav 2002;32:301-12.

46. Roy A. Characteristics of opiate dependent patients who attempt suicide. J Clin Psychiatry 2002;63:403-7.

47. Lopez P, Mosquera F, de Leon J, Gutierrez M, Ezcurra J, Ramirez F, Gonzalez-Pinto A.Suicide at- tempts inbipolar patients. J Clin Psychiatry 2001;62: 963-6.

48. Cooper J, Appleby L, Amos T. Life events preceding suicide by young people. Soc Psychiatry Psychiatr Epidemiol 2002;37:271-5.

49. Viilo K, Hakko H, Väisänen E, Räsänen P.Risk of suicide related to income level in mental illness. Letters. BMJ 2001;323:232.

50. Gunnell DJ, Peters TJ, Kammerling RM, Brooks J. Relation between parasuicide, suicide, psychiatry admissions and socioeconomic deprivation. BMJ 1995; 311:226-30.

51. WHO. Preventing Suicide. A resource for media professionls. Geneva: Department of Mental Health, World Health Organization; 2000. 